\title{
THE INTRAUTERINE DIAGNOSIS OF SKELETAL. DYSPLASIAS
}

Prof.Dr.Herbert J. Kaufmann, Berlin

The intrauterine recognition of skeletal dysplasias is served by two different methods.

Late diagnosis prior to a decision whether delivery should be vaginal or by Cesarian section depends on the radiologic recognition of the fetal skeletal dysplasia present and its individual prognosis.

Since a large number of skeletal dysplasias manifest at birth are lethal a fetal indication for Cesarian section will not improve the outlook for the baby. The differential diagnosis of such conditions on roentgenograms can therefor be the critical factor for the future obstetrical management of the mother.

If a woman has previously given birth to an infant with a skeletal dysplasia, particulary, if inherited as an autosomal dominant disorder, the management of further pregnancies must include early determination by ultrasound of the possibility of abnormal respective development. Accurate measurements of the relationship of the bitemporal diameter, the rumplength and its relationship to the length of the femur have already laid the groundwork for reliable diagnosis and this at a time, when therapeutic abortion can be safely performed.

The two methods outlined are complementary in order to establish a reliable diagnosis during early pregnancy. In any still-born or after an early demise of a new born baby there must be a full evaluation including $X$-ray studies to establish a correct diagnosis. This is the only way to supervise further pregnancies in a family affected by a genetically transmitted skeletal dysplasia of a potentially lethal type.

\author{
Prof.Dr.H.J.Kaufmann \\ Univ.-Kinderklinik \\ Heubnerweg 6 \\ D-1000 Berlin 19
}

